\title{
Performance Improvement of Phase-Based Correspondence Matching for Palmprint Recognition
}

\author{
Vincent Roux \\ Institut Supérieur d'électronique de Paris, \\ 28, rue Notre Dame des Champs, \\ 75006 Paris, France \\ vincent.roux@isep.fr
}

\author{
Shoichiro Aoyama, Koichi Ito, Takafumi Aoki \\ Graduate School of Information Sciences, \\ Tohoku University, \\ 6-6-05, Aramaki Aza Aoba, Aoba-ku, Sendai-shi, \\ 980-8579, Japan \\ aoyama@aoki.ecei.tohoku.ac.jp
}

\begin{abstract}
The use of phase-based correspondence matching for biometric recognition makes it possible to find corresponding point pairs between images having nonlinear deformation. On the other hand, the optimal recognition performance cannot be exhibited due to simple approaches for matching score calculation and reference point placement. This paper proposes two techniques to improve performance of phase-based correspondence matching for contactless palmprint recognition. First technique analyzes location of corresponding points and defines a new matching score. Second one selects location of reference points suggested by a Difference of Gaussians (DoG) filter. Through a set of experiments using CASIA contactless palmprint database, we demonstrate that the proposed techniques improve performance of phase-based correspondence matching and exhibit good performance compared with conventional palmprint recognition algorithms.
\end{abstract}

\section{Introduction}

Image deformation is one of the main problems in the field of biometric recognition and has to be solved to develop accurate recognition algorithms. This is remarkable for contactless biometric recognition systems, since images of a biometric trait acquired at the different timing have different deformation which is caused by pose changes even for the same person. Addressing the above problem, blockwise matching algorithms for biometric recognition have been proposed $[4,8,10]$.

The conventional algorithms robust against image deformation are broadly classified into two approaches: (i) feature matching such as Scale Invariant Feature Transform (SIFT) [7] and (ii) block matching such as probabilistic Deformation Models (PDM) and phase-based correspon- dence matching [12]. Morales et al. [8] proposed a contactless palmprint recognition algorithm using modified SIFT, which employs the preprocessing step to enhance image contrast and performs the matching validation process specially designed for palmprint matching after original SIFT matching. Ross et al. [10] proposed an ocular recognition algorithm using another version of modified SIFT, which employs the preprocessing step to enhance image contrast and introduces proximity and orientation constraint to the feature matching step. Ross et al. [10] also proposed an ocular recognition algorithm using PDM with OTSDF correlation filter. The use of PDM makes it possible to handle nonlinear deformation of images as translations of local block images. The matching score is calculated by the OTSDF correlation filter [13] is specially designed for block-wise deformation. Ito et al. [4] proposed a palmprint recognition algorithm using phase-based correspondence matching. The corresponding points on the input image are calculated from the reference points on the registered image by local block matching to handle nonlinear deformation of a palm.

Among the conventional algorithms mentioned above, phase-based correspondence matching is one of the powerful approaches for images with nonlinear deformation and has been successfully applied to some biometric recognition problems $[3,9,2]$. The process of matching score calculation is simpler than other algorithms such as modified SIFT, since the reference points are placed in a reticular pattern with constant spacing and the matching score is defined by the maximum peak value of average Band-Limited PhaseOnly Correlation (BLPOC) function. Hence, phase-based correspondence matching may allow us to improve performance of biometric recognition.

This paper proposes two techniques to improve performance of phase-based correspondence matching for contactless palmprint recognition. First technique analyzes location of corresponding points and defines a new 
matching score. Second one selects location of reference points suggested by a Difference of Gaussians (DoG) filter. Through a set of experiments using CASIA contactless palmprint database [1], we demonstrate that the proposed techniques improve performance of phase-based correspondence matching and exhibit good performance compared with conventional palmprint recognition algorithms.

\section{Palmprint Recognition Algorithm Using Phase-Based Correspondence Matching}

This section describes an outline of a palmprint recognition algorithm using phase-based correspondence matching proposed by Ito et al. [4]. This algorithm consists of (i) preprocessing, (ii) correspondence matching and (iii) matching score calculation. The step (i) extracts Region of Interest (ROI) from palmprint images using the method proposed by Zhang et al. [14]. The step (ii) finds points on the input image corresponding to reference points on the registered image using phase-based correspondence matching. The step (iii) calculates a matching score from the average BLPOC function. We describe the details of each step in the following.

\subsection{Preprocessing}

This step extracts a palmprint region to be matched from palmprint images. The method proposed in [14] is employed to extract the center part of a palm for accurate matching. This method uses gaps between fingers as reference points to define the palmprint region. At first, we apply the Gaussian low-pass filter to the input image and convert the smoothed image into the binary image by thresholding. Next, we extract boundaries of the binary image using a boundary tracking algorithm and determine the landmarks based on the extracted boundaries, where the landmarks are the bottom of gaps between index and middle fingers and between ring and little fingers. Then, we obtain the perpendicular bisector of the line segment between two landmarks to determine the centroid of the palmprint region. Finally, we extract the normalized palmprint region of fixed size, where the size of the region is $160 \times 160$ pixels. Fig. 1 shows an example of preprocessing.

\subsection{Correspondence Matching}

This step finds points on the input image corresponding to the reference points on the registered image using phasebased correspondence matching [12]. Phase-based correspondence matching used in the paper employs a coarseto-fine strategy using image pyramids for robust correspondence search and a translational displacement estimation method using BLPOC [5] for local block matching. Let $\boldsymbol{p}$ be a coordinate of a reference point in the registered image $I\left(n_{1}, n_{2}\right)$. We find a coordinate $\boldsymbol{q}$ in the input image $J\left(n_{1}, n_{2}\right)$ that corresponds to the reference point $\boldsymbol{p}$ in

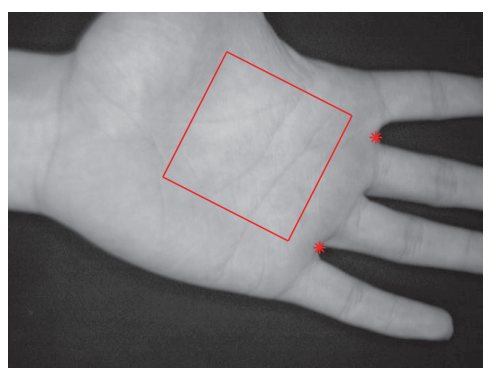

(a)

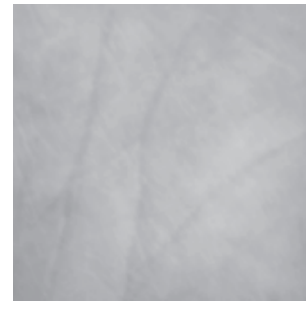

(b)
Figure 1. Preprocessing: (a) input image, and (b) extracted ROI.

$I\left(n_{1}, n_{2}\right)$. In this paper, $13 \times 13$ reference points are placed on the registered images $I\left(n_{1}, n_{2}\right)$ and then their corresponding points on the input image $J\left(n_{1}, n_{2}\right)$ are estimated by the following procedure. Note that we employ a 3-layer image pyramid in this paper.

Step 1: For $l=1,2$, create the $l$-th layer images $I^{l}\left(n_{1}, n_{2}\right)$ and $J^{l}\left(n_{1}, n_{2}\right)$, i.e., coarser versions of $I^{0}\left(n_{1}, n_{2}\right)$ (= $\left.I\left(n_{1}, n_{2}\right)\right)$ and $J^{0}\left(n_{1}, n_{2}\right)\left(=J\left(n_{1}, n_{2}\right)\right)$, recursively as follows:

$$
\begin{aligned}
& I^{l}\left(n_{1}, n_{2}\right)=\frac{1}{4} \sum_{i_{1}=0}^{1} \sum_{i_{2}=0}^{1} I^{l-1}\left(2 n_{1}+i_{1}, 2 n_{2}+i_{2}\right), \\
& J^{l}\left(n_{1}, n_{2}\right)=\frac{1}{4} \sum_{i_{1}=0}^{1} \sum_{i_{2}=0}^{1} J^{l-1}\left(2 n_{1}+i_{1}, 2 n_{2}+i_{2}\right) .
\end{aligned}
$$

Step 2: For $l=1,2$, calculate the coordinate $\boldsymbol{p}^{l}=\left(p_{1}^{l}, p_{2}^{l}\right)$ corresponding to the original reference point $\boldsymbol{p}^{0}(=\boldsymbol{p})$ as follows:

$$
\boldsymbol{p}^{l}=\left\lfloor\frac{1}{2} \boldsymbol{p}^{l-1}\right\rfloor=\left(\left\lfloor\frac{1}{2} p_{1}^{l-1}\right\rfloor,\left\lfloor\frac{1}{2} p_{2}^{l-1}\right\rfloor\right),
$$

where $\lfloor z\rfloor$ denotes the operation to round the element of $z$ to the nearest integer towards minus infinity.

Step 3: Estimate the displacement between $I^{2}\left(n_{1}, n_{2}\right)$ and $J^{2}\left(n_{1}, n_{2}\right)$ using BLPOC-based image matching. Let the estimated displacement vector be $\delta^{2}$. We assume that $\boldsymbol{q}^{2}=$ $p^{2}+\delta^{2}$ in the coarsest layer $(l=2)$. We set $l=1$.

Step 4: From the $l$-th layer images $I^{l}\left(n_{1}, n_{2}\right)$ and $J^{l}\left(n_{1}, n_{2}\right)$, extract two image blocks $f^{l}\left(n_{1}, n_{2}\right)$ and $g^{l}\left(n_{1}, n_{2}\right)$ with their centers on $\boldsymbol{p}^{l}$ and $2 \boldsymbol{q}^{l+1}$, respectively. The size of image blocks is $W \times W$ pixels. In this paper, we employ $W=48$.

Step 5: Estimate the displacement between $f^{l}\left(n_{1}, n_{2}\right)$ and $g^{l}\left(n_{1}, n_{2}\right)$ using BLPOC-based image matching. Let the estimated displacement vector be $\delta^{l}$. The $l$-th layer correspondence $\boldsymbol{q}^{l}$ is determined by

$$
\boldsymbol{q}^{l}=2 \boldsymbol{q}^{l+1}+\boldsymbol{\delta}^{l}
$$

Step 6: For $l=0$, repeat from Step 4 to Step 5. 


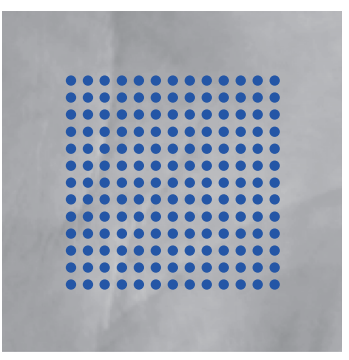

(a)

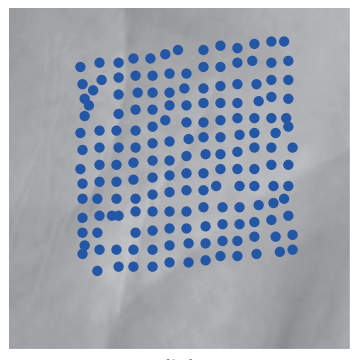

(b)
Figure 2. Correspondence matching between registered and input images: (a) registered image, and (b) input image.

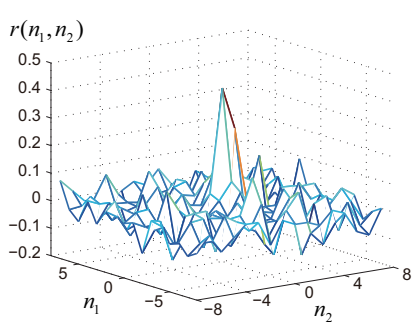

(a)

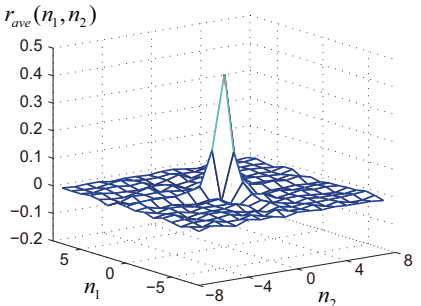

(b)
Figure 3. Matching score calculation: (a) BLPOC function between two local blocks, and (b) average BLPOC function.

We perform the above procedure for all the reference points on $I\left(n_{1}, n_{2}\right)$ and then obtain corresponding point pairs between $I\left(n_{1}, n_{2}\right)$ and $J\left(n_{1}, n_{2}\right)$. An example of correspondence point pairs obtained by phase-based correspondence matching is shown in Fig. 2. The location of corresponding points on the input image represents image deformation between the registered and input images.

\subsection{Matching Score Calculation}

This step calculates a matching score according to the corresponding point pairs obtained in the previous step. BLPOC functions between the local image blocks with their centers on corresponding point pairs are calculated. Then, the matching score is calculated as the highest peak value of the average BLPOC function obtained from a set of BLPOC functions. To take the average of a set of BLPOC functions, the PNR (Peak-to-Noise Ratio) of the BLPOC function can be improved as shown in Fig. 3.

\section{Performance Improvement Techniques}

This section presents performance improvement techniques for the palmprint recognition algorithm using phasebased correspondence matching [4].

We analyze the result of correspondence matching for genuine and imposter pairs. Fig. 4 is an example of reference points on the registered image and their corresponding points on the genuine and imposter input images. Loca-

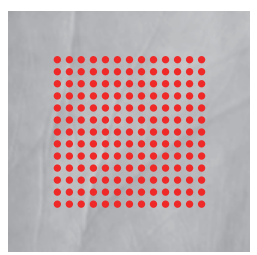

(a)

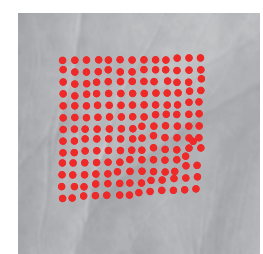

(b)

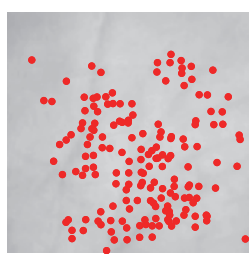

(c)
Figure 4. Example of correspondence matching: (a) reference points on the registered image, (b) corresponding points on the input image (genuine pair), and (c) corresponding points on the input image (imposter pair).
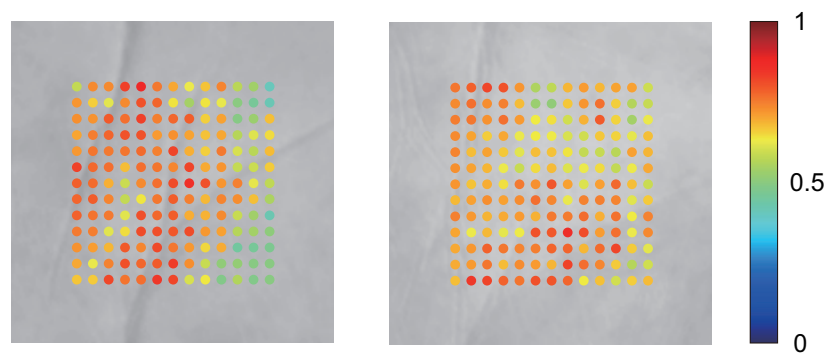

Figure 5. Maximum peak value of the BLPOC function for each local block image pair.

tion of corresponding points on the genuine input image is moved in accordance with deformation of image as shown in Fig. 4 (a) and (b). On the other hand, location of corresponding points on the imposter input image is randomly moved as shown in Fig. 4 (a) and (c), in other words, there is no relation between location of reference and corresponding points for the imposter pair. We observe that the structure of reference and corresponding points for the genuine pair has strong correlation, while that for imposter pair has no correlation. Hence, we can use geometric relation between reference and corresponding points to calculate a matching score.

We also analyze the maximum peak value of the BLPOC function for each local block image pair. Fig. 5 illustrates maps of peak values obtained in the corresponding matching process, where we take an average of maximum peak values calculated from 7 genuine pairs. We observe that the points having a higher peak value concentrate in regions around principal lines. The reference points are placed in a reticular pattern with constant spacing in the conventional algorithm [4]. However, this is not always the best for palmprint matching. Hence, we have to put reference points around principal lines to calculate an accurate matching score.

In accordance with the above discussions, we propose two performance improvement techniques for the palmprint recognition algorithm using phase-based correspondence matching. 


\subsection{Location-Based Matching Score Calculation}

In the conventional algorithm [4], the matching score is based on BLPOC function between local block images extracted from each corresponding point pair after correspondence matching. On the other hand, we propose a method to calculate a matching score directly from location of corresponding points.

Let us consider that we put $13 \times 13$ reference points with 8-pixel spacing on the registered image and find their corresponding points on the input image using phase-based correspondence matching. We obtain correspondence between the registered and input images after correspondence matching as shown in Fig. 6 (a) and (b). We define a graph $F_{r}(V, E)$ on the registered image by connecting adjacent reference points as shown in Fig. 6 (a), where $V$ is a set of nodes and $E$ is a set of edges. Using the same connections in $F_{r}$, we also define a graph $F_{i}(V, E)$ on the input image as shown in Fig. 6 (b). We calculate the distance of each corresponding edge pair between $E\left(F_{r}\right)$ and $E\left(F_{i}\right)$ and then make a histogram $H(x)$ of edge distance as shown in Fig. 6 (c), where $x$ is a distance.

If we obtain accurate corresponding points for a genuine pair, the resultant histogram concentrates in a certain distance $x$ as shown in Fig. 6 (c), since the relation between reference and corresponding points can be represented by a geometric transformation model. On the other hand, as for an imposter pair, the resultant histogram is widely distributed as shown in Fig. 7 (c), since location of the corresponding points is randomly moved as shown in Fig. 7 (b).

We estimate the shape of the histogram $H(x)$ by fitting the Gaussian function $G(x)$ as

$$
G(x)=\frac{A}{\sqrt{2 \pi} \sigma} \exp \left(-\frac{(x-\mu)^{2}}{2 \sigma^{2}}\right),
$$

where $\sigma$ is a standard deviation of the Gaussian function, $\mu$ is a mean of the Gaussian function and $A$ is a parameter. When the interval between adjacent reference points is 8 pixels, we optimize parameters $A, \sigma$ and $\mu$ within the range $2 \leq x \leq 14$. We calculate the matching score as the maximum value of the Gaussian function defined by $A /(\sqrt{2 \pi} \sigma)$.

Fig. 8 shows an example of matching a genuine pair having a low score and Fig. 9 shows an example of matching an imposter pair having a high score in the conventional algorithm [4]. As a result, our location-based matching score exhibits better matching performance than using maximum peak value of average BLPOC function, since the matching score of the genuine pair is higher than that of imposter pair. In addition, the matching scores calculated with the conventional and proposed algorithms play a complemental role each other. Hence, it is expected that matching performance can be improved by fusing the two matching scores.

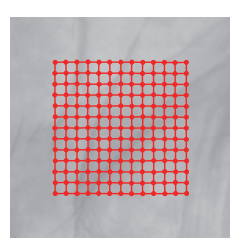

(a)

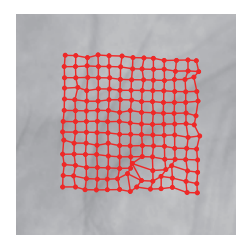

(b)

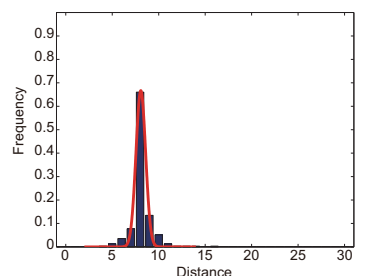

(c)
Figure 6. Location-based matching score calculation for a genuine pair: (a) reference points on the registered image and a graph $F_{r}$, (b) corresponding points on the input image and a graph $F_{i}$, and (c) histogram $H(x)$ of edge distance between $F_{r}$ and $F_{i}$.

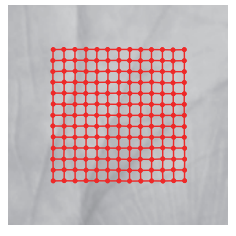

(a)

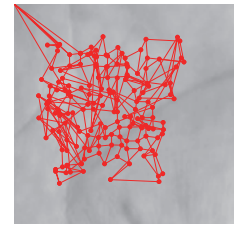

(b)

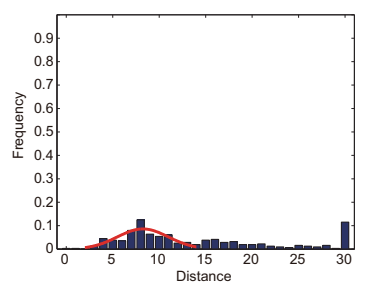

(c)
Figure 7. Location-based matching score calculation for an imposter pair: (a) reference points on the registered image and a graph $F_{r}$, (b) corresponding points on the input image and a graph $F_{i}$, and (c) histogram $H(x)$ of edge distance between $F_{r}$ and $F_{i}$.

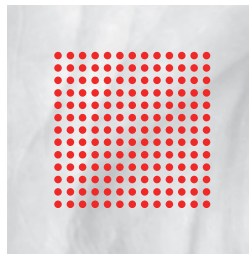

(a)

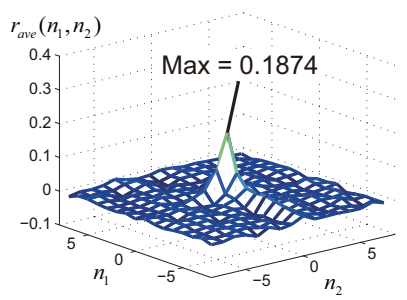

(c)

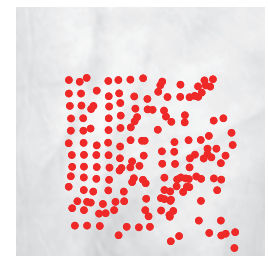

(b)

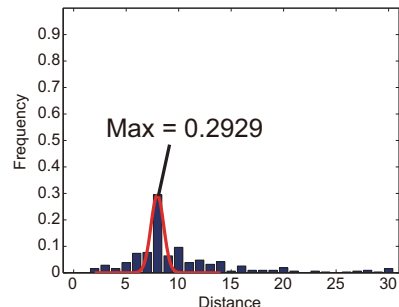

(d)
Figure 8. Example of genuine matching: (a) reference points on the registered image, (b) corresponding points on the input image, (c) average BLPOC function, and (d) histogram of edge distance.

\subsection{DoG-Based Reference Point Selection}

The reference points are placed on the registered image in a reticular pattern with the constant spacing in the conventional algorithm. On the other hand, we consider to select a set of reference points around principal lines by using 


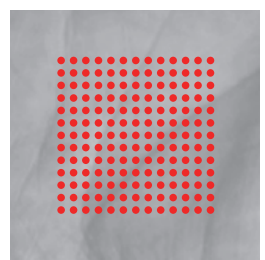

(a)

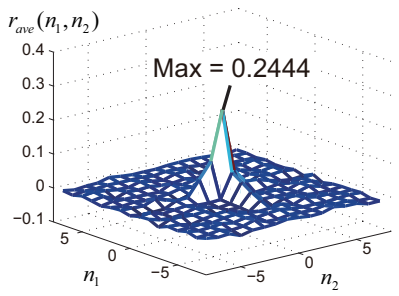

(c)

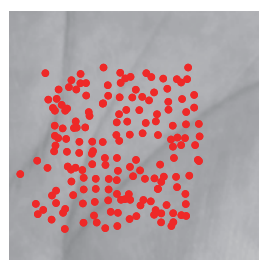

(b)

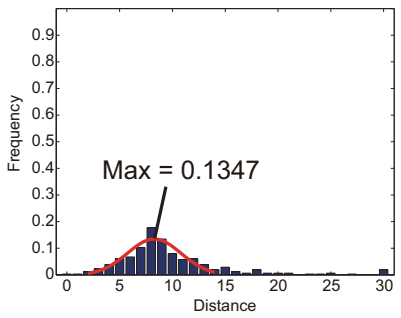

(d)
Figure 9. Example of imposter matching: (a) reference points on the registered image, (b) corresponding points on the input image, (c) average BLPOC function, and (d) histogram of edge distance.

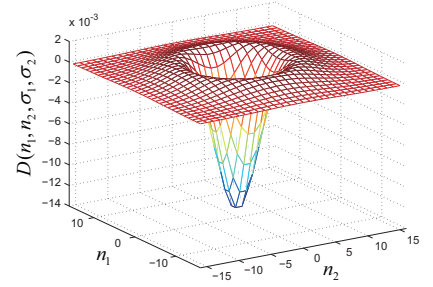

Figure 10. Example of the function shape of a DoG filter $D\left(n_{1}, n_{2}, \sigma_{1}, \sigma_{2}\right)$.

a Difference of Gaussians (DoG) filter.

The DoG filter $D\left(n_{1}, n_{2}, \sigma_{1}, \sigma_{2}\right)$ is defined by the difference between outputs from 2D Gaussian filters with different standard deviations $\sigma_{1}$ and $\sigma_{2}$, and is given by

$$
D\left(n_{1}, n_{2}, \sigma_{1}, \sigma_{2}\right)=G^{\prime}\left(n_{1}, n_{2}, \sigma_{1}\right)-G^{\prime}\left(n_{1}, n_{2}, \sigma_{2}\right),
$$

where

$$
G^{\prime}\left(n_{1}, n_{2}, \sigma\right)=\frac{1}{2 \pi \sigma^{2}} \exp \left(-\frac{n_{1}^{2}+n_{2}^{2}}{2 \sigma^{2}}\right) .
$$

In this paper, we employ $\sigma_{1}=0.65$ and $\sigma_{2}=0.2$. Fig. 10 shows an example of the function shape of a DoG filter $D\left(n_{1}, n_{2}, \sigma_{1}, \sigma_{2}\right)$.

The procedure of DoG-based reference point selection is summarized as follows:

Step 1: Apply the DoG filter $D\left(n_{1}, n_{2}, \sigma_{1}, \sigma_{2}\right)$ to the registered image (Fig. 11 (a)) and obtain the filter response as shown in Fig. 11 (b).

Step 2: Find the point having maximum value in the filter response as a reference point for correspondence matching

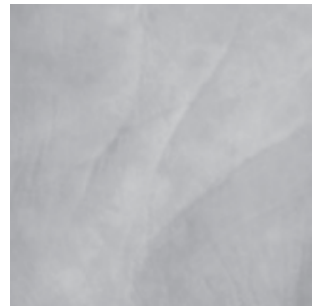

(a)

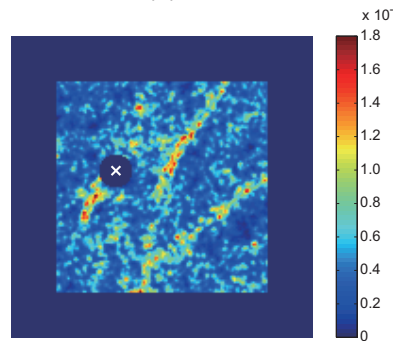

(c)

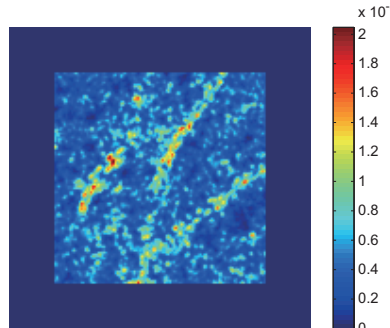

(b)

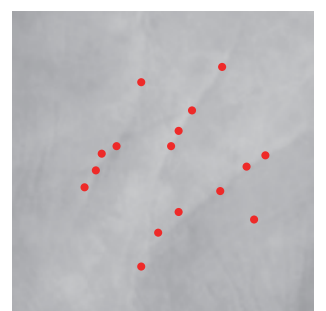

(d)
Figure 11. DoG-based reference point selection: (a) registered image, (b) filter response, (c) point having maximum value in the filter response, and (d) selected reference points.

and fill the circular region with 8-pixel radius centered at the point with zero as shown in Fig. 11 (c).

Step 3: Repeat Step 2 until the number of reference points satisfies the requirement.

Fig. 11 (d) shows an example when 16 reference points are selected by the above procedure. Using the DoG filter, we can put the reference points on principal lines.

Fig. 12 shows an example of matching a genuine pair. The reference points are placed on the registered image in a reticular pattern as well as the conventional algorithm as shown in Fig. 12 (a)-(c), while the reference points are selected by the DoG-based reference point selection procedure as shown in Fig. 12 (d)-(f). The maximum peak value of the average BLPOC function for the proposed algorithm is higher than that for the conventional algorithm. Fig. 13 shows an example of matching an imposter pair. The maximum peak value of the average BLPOC function for the proposed algorithm is comparable with that for the conventional algorithm. As observed above, the use of the DoGbased reference point selection technique makes it possible to improve the matching score for genuine pairs.

\section{Experiments and Discussion}

We evaluate performance of proposed techniques mentioned in Sect. 3.1 and 3.2 for the palmprint recognition algorithm using phase-based correspondence matching [4]. In this paper, we use CASIA Palmprint database [1] for experiments. The CASIA Palmprint database consists of 5,239 contactless palmprint images with left and right palm of 301 subjects. Fig. 14 shows examples of images in the CA- 


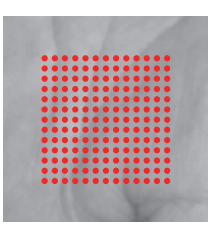

(a)

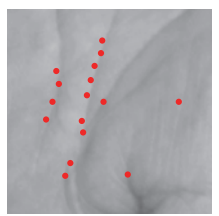

(d)

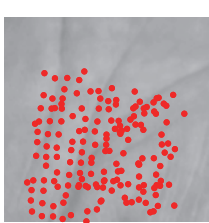

(b)

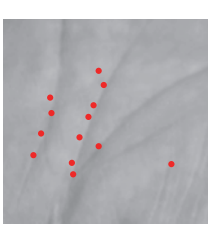

(e)

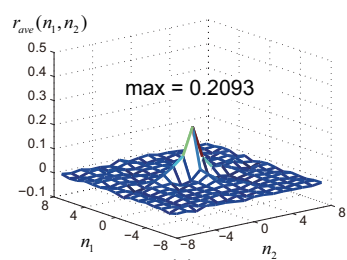

(c)

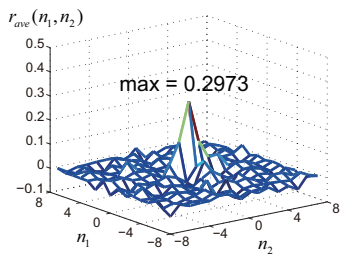

Figure 12. Example of matching a genuine pair: (a) registered image and reference points placed in a reticular pattern, (b) input image and corresponding points, (c) average BLPOC function, (d) registered image and reference points selected by the proposed technique, (e) input image and corresponding points, and (f) average BLPOC function.

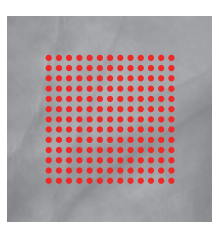

(a)

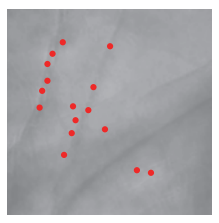

(d)

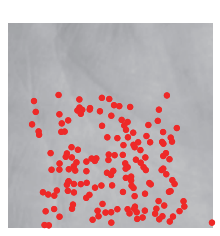

(b)

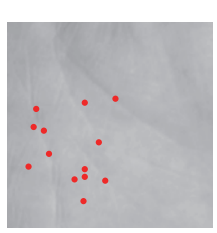

(e)

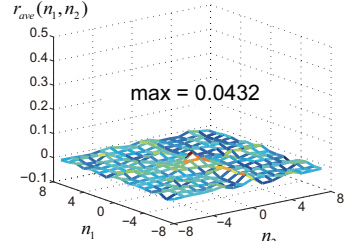

(c)

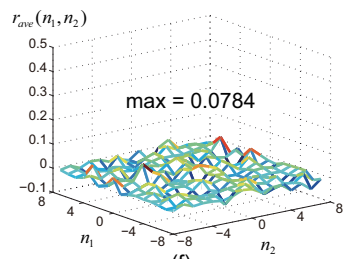

(f)
Figure 13. Example of matching an imposter pair: (a) registered image and reference points placed in a reticular pattern, (b) input image and corresponding points, (c) average BLPOC function, (d) registered image and reference points selected by the proposed technique, (e) input image and corresponding points, and (f) average BLPOC function.

SIA database. Each column illustrates ROI images from the same subject. As observed in this figure, images have large deformation, since the images are acquired under contactless conditions.

The performance of the biometrics-based verification system is evaluated by the Receiver Operating Characteristic (ROC) curve, which illustrates the False Rejection Rate (FRR) against the False Acceptance Rate (FAR) at different thresholds on the matching score. We first evaluate the FRR for all the possible combinations of genuine attempts; the number of attempts is 20,584 . Next, we evaluate the FAR

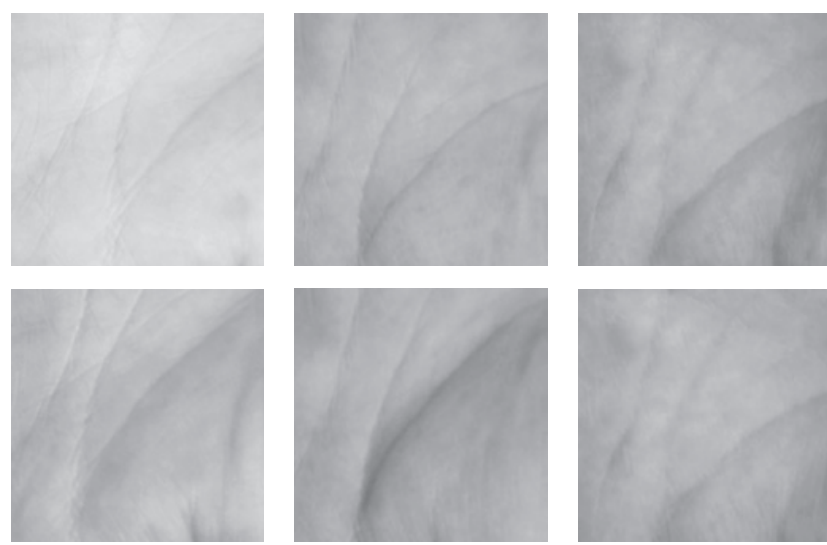

Figure 14. Examples of extracted ROI from images in the CASIA Palmprint database, where each column illustrates ROI images from the same subject.

for all the possible combinations of imposter attempts; the number of attempts is $13,700,357$. The performance is also evaluated by the Equal Error Rate (EER), which is defined as the error rate where the FRR and the FAR are equal.

We compare recognition performance of the conventional algorithms such as CompCode [6], Ordinal Code [11], SMCC [15], and phase-based correspondence matching [4] with that of the proposed algorithms with improvement techniques. In the following, we denote the palmprint recognition algorithm using phase-based correspondence matching [4] as "Original," location-based matching score calculation as "Location" and DoG-based reference point selection as "DoG." The number of reference points for phase-based correspondence matching is $13 \times 13$ for Original and Location. We consider to improve recognition performance by combining the matching scores from Original and Location. In this paper, average of Original and Location is used as the combined matching score. We select 16, 32, 64 and 100 reference points using DoG in the experiments. The parameter of BLPOC function is $K_{1} / M_{1}=K_{2} / M_{2}=0.33$ in the experiments.

Fig. 15 shows ROC curves of the conventional and proposed palmprint recognition algorithms and Table 1 summarizes their EERs, where the EERs for the conventional algorithms are referred from Ref. [15]. EERs of the algorithms using phase-based correspondence matching are lower than those of the conventional algorithms such as CompCode, Ordinal Code, and SMCC. The EER of Location is comparable with that of Original. Combining matching scores of Original and Location using the simple sum rule, EER can be significantly improved, since Original and Location play a complemental role in palmprint image matching. The EERs of DoG except for 16 reference points are lower than that of Original, since reliable local block images to calculate the average BLPOC function are selected 


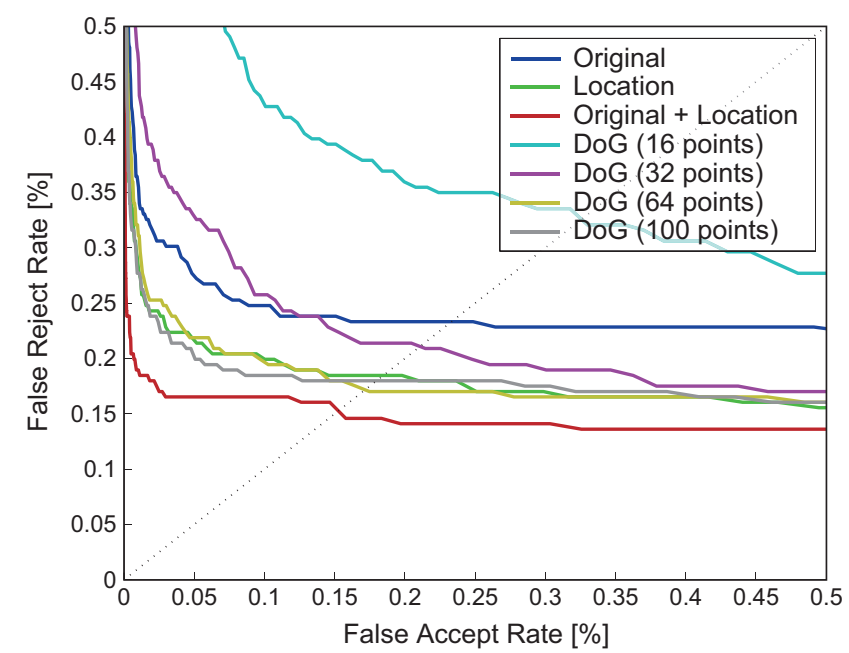

Figure 15. ROC curves of palmprint recognition algorithms.

Table 1. EERs [\%] of palmprint recognition algorithms.

\begin{tabular}{|c|c|c|}
\hline \multicolumn{2}{|l|}{ Algorithm } & $\operatorname{EER}[\%]$ \\
\hline \multicolumn{2}{|l|}{ CompCode [15] } & 0.55 \\
\hline \multicolumn{2}{|c|}{ Ordinal Code [15] } & 0.84 \\
\hline \multicolumn{2}{|c|}{ SMCC [15] } & 0.48 \\
\hline \multirow{7}{*}{$\begin{array}{l}\text { Phase-based } \\
\text { correspondence } \\
\text { matching }\end{array}$} & Original & 0.23 \\
\hline & Location & 0.18 \\
\hline & Original + Location & 0.15 \\
\hline & DoG (16 points) & 0.33 \\
\hline & DoG (32 points) & 0.21 \\
\hline & DoG (64 points) & 0.17 \\
\hline & DoG (100 points) & 0.18 \\
\hline
\end{tabular}

by DoG-based reference point selection in advance.

We evaluate the computation time for each proposed algorithm. We implement these algorithms using MATLAB and measure their computation time using Intel Xeon X5690 (3.46GHz) with a single thread running. Table 2 summarizes computation time of Original, Location and DoG. Location is faster than Original, since Location does not calculate BLPOC functions for all the local image pairs. DoG is significant faster than Original, since the number of reference points in DoG is less than that in Original. The proposed techniques improve recognition performance as well as computation time of the palmprint recognition algorithm using phase-based correspondence matching.

\section{Conclusion}

This paper has proposed two performance improvement techniques for the palmprint recognition algorithm using phase-based correspondence matching. First technique analyzes location of corresponding points and defines a new matching score. Second one selects location of reference
Table 2. Computation time [ms] of proposed algorithms.

\begin{tabular}{l|c}
\hline Algorithm & Time $[\mathrm{ms}]$ \\
\hline Original & 380 \\
Location & 349 \\
Original + Location & 392 \\
DoG (16 points) & 59 \\
DoG (32 points) & 93 \\
DoG (64 points) & 168 \\
DoG (100 points) & 252 \\
\hline
\end{tabular}

points suggested by a Difference of Gaussians (DoG) filter. Through a set of experiments using CASIA contactless palmprint database [1], we demonstrate that the proposed techniques improve performance of phase-based correspondence matching and exhibit good performance compared with conventional palmprint recognition algorithms. We focus on contactless palmprint recognition through the paper. The discussion could be applied to biometric recognition algorithms using phase-based correspondence matching for other biometric traits such as face, iris, etc.

\section{References}

[1] CASIA palmprint database. http://www.cbsr.ia. ac.cn/english/PalmprintDatabases.asp. 2, 5, 7

[2] S. Aoyama, K. Ito, and T. Aoki. A finger-knuckle-print recognition algorithm using phase-based local block matching. Information Sciences, 268(1):53-64, June 2014. 1

[3] K. Ito, T. Aoki, T. Hosoi, and K. Kobayashi. Face recognition using phase-based correspondence matching. Proc. Int'l Conf. Automatic Face and Gesture Recognition, pages 173178, Mar. 2011. 1

[4] K. Ito, S. Iitsuka, and T. Aoki. A palmprint recognition algorithm using phase-based correspondence matching. Proc. Int'l Conf. Image Processing, pages 1977-1980, Nov. 2009. $1,2,3,4,5,6$

[5] K. Ito, H. Nakajima, K. Kobayashi, T. Aoki, and T. Higuchi. A fingerprint matching algorithm using phase-only correlation. IEICE Trans. Fundamentals, E87-A(3):682-691, Mar. 2004. 2

[6] A.-K. Kong and D. Zhang. Competitive coding scheme for palmprint verification. Proc. Int'l Conf. Pattern Recognition, 1:520-523, Dec. 2004. 6

[7] D. Lowe. Distinctive image features from scale-invariant keypoints. Int'l J. Computer Vision, 60(2):91-110, Jan. 2004. 1

[8] A. Morales, M. Ferrer, and A. Kumar. Towards contactless palmprint authentication. IET Computer Vision, 5(6):407416, 2011. 1

[9] H. Ota, S. Aoyama, R. Watanabe, K. Ito, Y. Miyake, and T. Aoki. Implementation and evaluation of a remote authentication system using touchless palmprint recognition. Multimedia Systems, (2):117-129, Mar. 2013. 1 
[10] A. Ross, R. Jillela, J. Smereka, V. Boddeti, B. Kumar, R. Barnard, X. Hu, P. Pauca, and R. Plemmons. Matching highly non-ideal ocualr images: An information fusion approach. Proc. Int'l Conf. Biometrics, pages 446-453, 2012. 1

[11] Z. Sun, T. Tan, Y. Wang, and S. Li. Ordinal palmprint represention for personal identification. Proc. IEEE Computer Society Conf. Computer Vision and Pattern Recognition, 1:279-284, June 2005. 6

[12] K. Takita, M. A. Muquit, T. Aoki, and T. Higuchi. A subpixel correspondence search technique for computer vision applications. IEICE Trans. Fundamentals, E87-A(8):19131923, Aug. 2004. 1, 2

[13] J. Thornton, M. Savvides, and B. Kumar. A Bayesian approach to deformed pattern matching of iris images. IEEE Trans. Pattern Analysis and Machine Intelligence, 29(4):596-606, Apr. 2007. 1

[14] D. Zhang, W.-K. Kong, J. You, and M. Wong. Online palmprint identification. IEEE Trans. Pattern Analysis and Machine Intelligence, 25(9):1041-1050, Sept. 2003. 2

[15] W. Zuo, Z. Lin, Z. Guo, and D. Zhang. The multiscale competitive code via sparse representation for palmprint verification. Proc. IEEE Computer Society Conf. Computer Vision and Pattern Recognition, pages 2265-2272, June 2010. 6, 7 